\title{
Wirken Z-Substanzen auch antidepressiv?
}

\author{
Eine Metaanalyse legt nahe, dass die Gabe einer Z-Substanz die Wirkung von Antidepressiva \\ boostern könnte. Allerdings lohnt es sich, die Effektstärke einmal genauer anzusehen.
}

_Eine Metaanalyse sollte untersuchen, ob die bei Schlafstörungen indizierten Z-Substanzen Zolpidem und Zopiclon selbst antidepressive Effekte entfalten, wenn sie mit Antidepressiva kombiniert werden. In den identifizierten sechs Studien erhielten die insgemsamt 2.089 Patienten zusätzlich zu selektiven Serotonin-Wiederaufnahmehemmern oder Venlafaxin entweder eine Z-Substanz oder Placebo.

Primärer Endpunkt waren die $\mathrm{Re}$ missionsrate und die Häufigkeit des Absetzens. Sekundäre Endpunkte waren die Response-Raten, die Besserung auf der Hamilton Depression Scale (HAMD), das Absetzen wegen Ineffektivität sowie
Nebenwirkungen. Die mittlere Studiendauer betrug 10,5 Wochen.

Mit einer Z-Substanz ergab sich eine bessere Remissionsrate (relatives Risiko: 0,85 , Number needed to treat: 10) und ein kleiner Vorteil bei den HAMD-Werten (Effektstärke: 0,23). Response- und Absetzraten waren nicht unterschiedlich. Die Add-on-Therapie mit einer Z-Substanz führte zu mehr Nebenwirkungen.

- Kishi Tet al. Efficacy and tolerability of Z-drug adjunction to antidepressant treatment for major depressive disorder: a systematic review and meta-analysis of randomized controlled trials. Eur Arch Psychiatry Clin Neurosci. 2017;267:149-61

\section{KOMMENTAR}

Auch wenn die Analyse gut gemacht ist, zeigen die ermittelten Effektstärken, dass eine zusätzliche Z-Substanz-Gabe nur kleine antidepressive Effekte im Vergleich zu Placebo aufweist. Dazu passt die in diesem Kontext hoch erscheinende Number needed to treat von 10.

Da Abhängigkeitsentwicklungen unter Z-Substanzen beschrieben wurden, muss die kleine Überlegenheit in den antidepressiven Effekten gegen vermehrte $\mathrm{Ne}$ benwirkungen abgewogen werden. Aus meiner Sicht bleibt es dabei, dass ebenso wie bei Benzodiazepinen auch Z-Substanzen nur vorübergehend gegeben werden sollten und Nebenwirkungen und Abhängigkeitsentwicklung engmaschig kontrolliert werden müssen.

Prof. Dr. med. K. Lieb

\section{Hier steht eine Anzeige.}

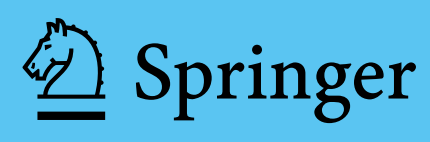

